\title{
Lack of antibody affinity maturation due to poor Toll-like receptor stimulation leads to enhanced respiratory syncytial virus disease
}

\author{
Maria Florencia Delgado ${ }^{1}$, Silvina Coviello ${ }^{1}$, A Clara Monsalvo ${ }^{1}$, Guillermina A Melendi ${ }^{1,2}$, \\ Johanna Zea Hernandez ${ }^{1,2}$, Juan P Batalle ${ }^{1}$, Leandro Diaz ${ }^{1}$, Alfonsina Trento ${ }^{3}$, Herng-Yu Chang ${ }^{4}$, \\ Wayne Mitzner ${ }^{4}$, Jeffrey Ravetch ${ }^{5}$, José A Melero ${ }^{3}$, Pablo M Irusta ${ }^{1,6}$ \& Fernando P Polack ${ }^{1,2,7,8}$
}

\begin{abstract}
Respiratory syncytial virus (RSV) is a leading cause of hospitalization in infants. A formalin-inactivated RSV vaccine was used to immunize children and elicited nonprotective, pathogenic antibody. Immunized infants experienced increased morbidity after subsequent RSV exposure. No vaccine has been licensed since that time. A widely accepted hypothesis attributed the vaccine failure to formalin disruption of protective antigens. Here we show that the lack of protection was not due to alterations caused by formalin but instead to low antibody avidity for protective epitopes. Lack of antibody affinity maturation followed poor Toll-like receptor (TLR) stimulation. This study explains why the inactivated RSV vaccine did not protect the children and consequently led to severe disease, hampering vaccine development for $\mathbf{4 2}$ years. It also suggests that inactivated RSV vaccines may be rendered safe and effective by inclusion of TLR agonists in their formulation, and it identifies affinity maturation as a key factor for the safe immunization of infants.
\end{abstract}

Approximately $50 \%$ of infants are infected with RSV during their first year of life ${ }^{1,2}$. Hospitalization rates for RSV in the United States have risen by $239 \%$ in recent decades ${ }^{3}$, and there is still no vaccine licensed against the virus.

A major obstacle to vaccine development has been the enhanced respiratory disease (ERD) that affected children immunized with a formalin-inactivated vaccine against RSV (FIRSV) in the $1960 \mathrm{~s}^{4}$. The vaccine was immunogenic, but it elicited a nonprotective antibody response ${ }^{4}$. Immunized children exposed to RSV in the community and seronegative for the virus before vaccination experienced an increase in the severity of lung disease ${ }^{4}$. Furthermore, two immunized infants died as toddlers upon subsequent RSV infection ${ }^{4}$. High titers of RSV were recovered from their lungs ${ }^{4}$. The main clinical manifestations in children with ERD were bronchoconstriction and a severe pneumonia ${ }^{4}$, and the disease was associated with an excess in peribronchiolar eosinophils ${ }^{4}$ and nonprotective antibody complexed with virus deposited in affected tissue ${ }^{5}$.

Even though the immune phenotype of ERD has been extensively characterized $^{6-12}$, the most pressing question about the pathogenesis of ERD is why antibodies elicited by FIRSV failed to protect against $\mathrm{RSV}$-a protective response would have prevented ERD. A widely accepted theory ascribes the lack of protection to formalin disruption of key epitopes during vaccine inactivation ${ }^{7,13,14}$. But the inability of other nonreplicating RSV vaccines to elicit protective antibody and their propensity to trigger aberrant immune manifestations (for example, a $\mathrm{T}$ helper type $2\left(\mathrm{~T}_{\mathrm{H}} 2\right)$ bias $\left.^{6,10-12}\right)$, suggest that ERD pathogenesis cannot be attributed to solely poor preservation of specific antigens. In fact, high immunoassay to neutralization ratios in the nonprotective antibody response elicited by FIRSV and other nonreplicating RSV immunogens ${ }^{11,12}$ and the relationship between better binding strength for the protective $\mathrm{F}$ protein and neutralizing capacity in humanized RSV monoclonal antibodies ${ }^{15}$ suggest that affinity maturation may be essential in protection against RSV.

Understanding the mechanisms that led to the production of nonprotective, pathogenic antibody in response to FIRSV and the requirements for eliciting protective antibody against RSV is crucial for the development of safe vaccines to protect infants. Drawing from the observations described above, we tested the hypothesis that maturation of avidity has a key role in protective responses against RSV and that production of antibody of low avidity for the virus was the main cause for the vaccine failure and consequent ERD development in affected children.

1INFANT Foundation, Gavilan 94, Buenos Aires 1406, Argentina. 2Department of Pediatrics, School of Medicine, Johns Hopkins University, 200 North Wolfe Street, Baltimore, Maryland 21205, USA. ${ }^{3}$ Instituto de Salud Carlos III and CIBER de Enfermedades Respiratorias, Pozuelo km.2, Majadahonda, Madrid 28220, Spain. ${ }^{4}$ Department of Environmental Health, Bloomberg School of Public Health, Johns Hopkins University, 615 North Wolfe Street, Baltimore, Maryland 21205 , USA. ${ }^{5}$ Rockefeller University, 1230 York Avenue, New York, New York 10065, USA. ${ }^{6}$ Department of Human Science, Georgetown University, 3700 Reservoir Road, Washington, DC 20007, USA. ${ }^{7}$ Department of Molecular Microbiology and Immunology and ${ }^{8}$ Department of International Health, Bloomberg School of Public Health, Johns Hopkins University, 615 North Wolfe Street, Baltimore, Maryland, 21205, USA. Correspondence should be addressed to F.P.P. (fpolack@jhsph.edu). 
Figure 1 Nonreplicating vaccines against RSV prime for ERD. (a) AHR $7 \mathrm{~d}$ after RSV challenge in previously immunized BALB/C mice. AHR to acetylcholine challenge is defined by the time-integrated rise in peak airway pressure. Results are means \pm s.e.m. of 8-11 mice per group and are representative of two independent experiments. For FIRSV, $P<0.05$ versus placebo or RSV. APTI, airway pressure time index. (b) Pulmonary histopathology $7 \mathrm{~d}$ after RSV challenge in mice that had received the indicated preimmunizations. H\&E staining shows peribronchiolar pneumonia, hematoxylin and Congo Red (H\&CR) staining shows pulmonary eosinophlia and periodic acid-Schiff (PAS) staining shows enhanced bronchiolar mucus production in FIRSV, UVRSV and PFP recipients. Scale bar for H\&E and PAS, $100 \mu \mathrm{m}$; scale bar for H\&CR, $25 \mu \mathrm{m}$. The boxes in H\&CR images are details of eosinophil lung infiltation. (c) Pulmonary eosinophils per 40X field counts in recipients of FIRSV, UVRSV, PFP, RSV and placebo. Results are means \pm s.e.m. of six to ten mice per group and are representative of two independent experiments. For FIRSV, $P<0.05$ versus RSV, placebo and PFP. (d) Lung viral titers $4 \mathrm{~d}$ after RSV challenge in recipients of inactivated vaccines, RSV or placebo. Results are means \pm s.e.m. of six mice per group. For FIRSV, $P<0.05$ versus RSV.

\section{RESULTS}

\section{Nonreplicating vaccines against RSV prime for ERD}

The manifestations of ERD in animal models include airways hyperresponsiveness (AHR), severe pneumonia with pulmonary eosinophilia and deficient protective antibody production with recovery of RSV from the lungs ${ }^{5,9-12}$. To determine whether various nonreplicating vaccines can prime for ERD, we inoculated mice in the footpad with FIRSV, RSV inactivated with ultraviolet light (UVRSV) or purified fusion protein (PFP). The fusion (F) protein is the main neutralizing antigen in $\mathrm{RSV}^{1}$. Control mice were inoculated with wild-type (WT) RSV intranasally (i.n.), which confers protection against the virus ${ }^{1}$. An additional control group received a footpad dose of supernatant fluid from lysate of Hep-2 cells as a placebo (Fig. 1).

All mice were challenged i.n. with WT RSV $60 \mathrm{~d}$ after vaccination, and those immunized with FIRSV, UVRSV or PFP had increased AHR compared to mice protected by a previous WT RSV infection or mice immunized with placebo (Fig. 1a). The three groups immunized with nonreplicating vaccines also had severe perivascular and peribronchiolar pneumonia, whereas WT RSV and placebo recipients had mild lung infiltration (Fig. 1b and Supplementary Methods online). In addition, mice primed with nonreplicating immunogens had eosinophilic infiltration and more mucus in the lungs than mice from control groups (Fig. 1b,c). Lung virus titers in FIRSV, UVRSV and PFP vaccinees were similar to those in placebo recipients (Fig. 1d). No virus was recovered from the lungs of mice immunized with WT RSV (Fig. 1d). These findings demonstrate that various nonreplicating vaccines can prime for ERD.

Nonreplicating vaccines elicit nonprotective, low avidity antibody The protective role of neutralizing antibody against RSV is well established $^{16,17}$. In fact, unlike cytotoxic $\mathrm{T}$ lymphocytes ${ }^{18}$, protective antibodies prevent RSV replication in the lungs upon viral re-challenge (Supplementary Fig. 1 online).

Because inactivated vaccines failed to protect against RSV, we compared the antibody response elicited by inactivated and live vaccines against the neutralizing $\mathrm{F}$ protein. Both FIRSV and UVRSV
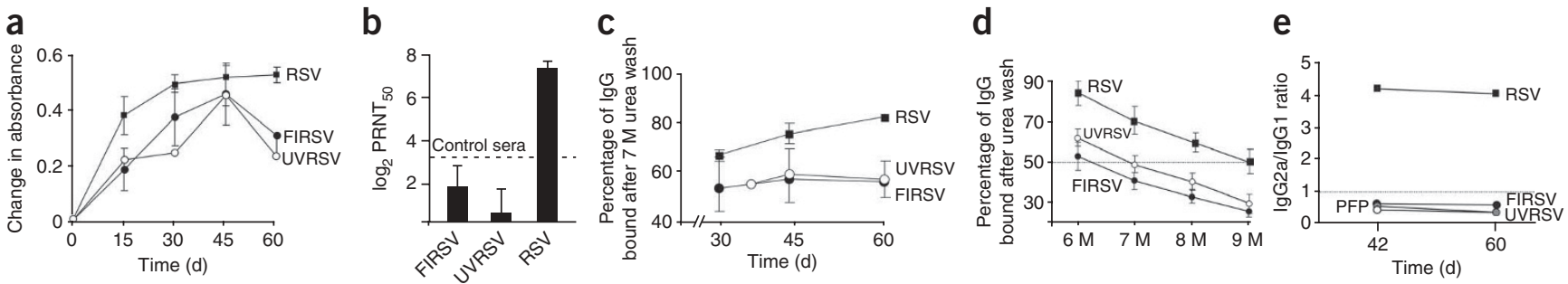

Figure 2 Nonreplicating vaccine elicits non-protective, low avidity antibody. (a) IgG antibody responses against the RSV F protein determined by immunoassay from sera of FIRSV, UVRSV and RSV recipients. For FIRSV, $P<0.05$ compared with RSV. (b) RSV-specific neutralization, as measured by $50 \%$ plaque reduction $\left(\mathrm{PRNT}_{50}\right.$ ) on day 45 after immunization. For $\mathbf{a}$ and $\mathbf{b}$, results are means \pm s.e.m. of five to eight mice per group and are representative of three independent experiments. For FIRSV, $P=0.003$ compared to RSV. For UVRSV, $P=0.002$ compared to RSV. (c) Determination of IgG avidity against RSV F after a $7 \mathrm{M}$ urea wash in sera from preimmunized BALB/c mice. For FIRSV, $P<0.05$ compared to RSV. Results are means \pm s.e.m. of six to ten mice per group and are representative of three independent experiments. (d) Determination of IgG avidity against RSV F protein in sera from preimmunized mice on day 45 after immunization. $50 \%$ avidity for FIRSV $P<0.001$ versus RSV. For UVRSV, $P<0.001$ versus RSV. (e) IgG $2 a /$ lgG 1 ratio against RSV F protein in sera from preimmunized mice. Results are representative of two independent experiments. 

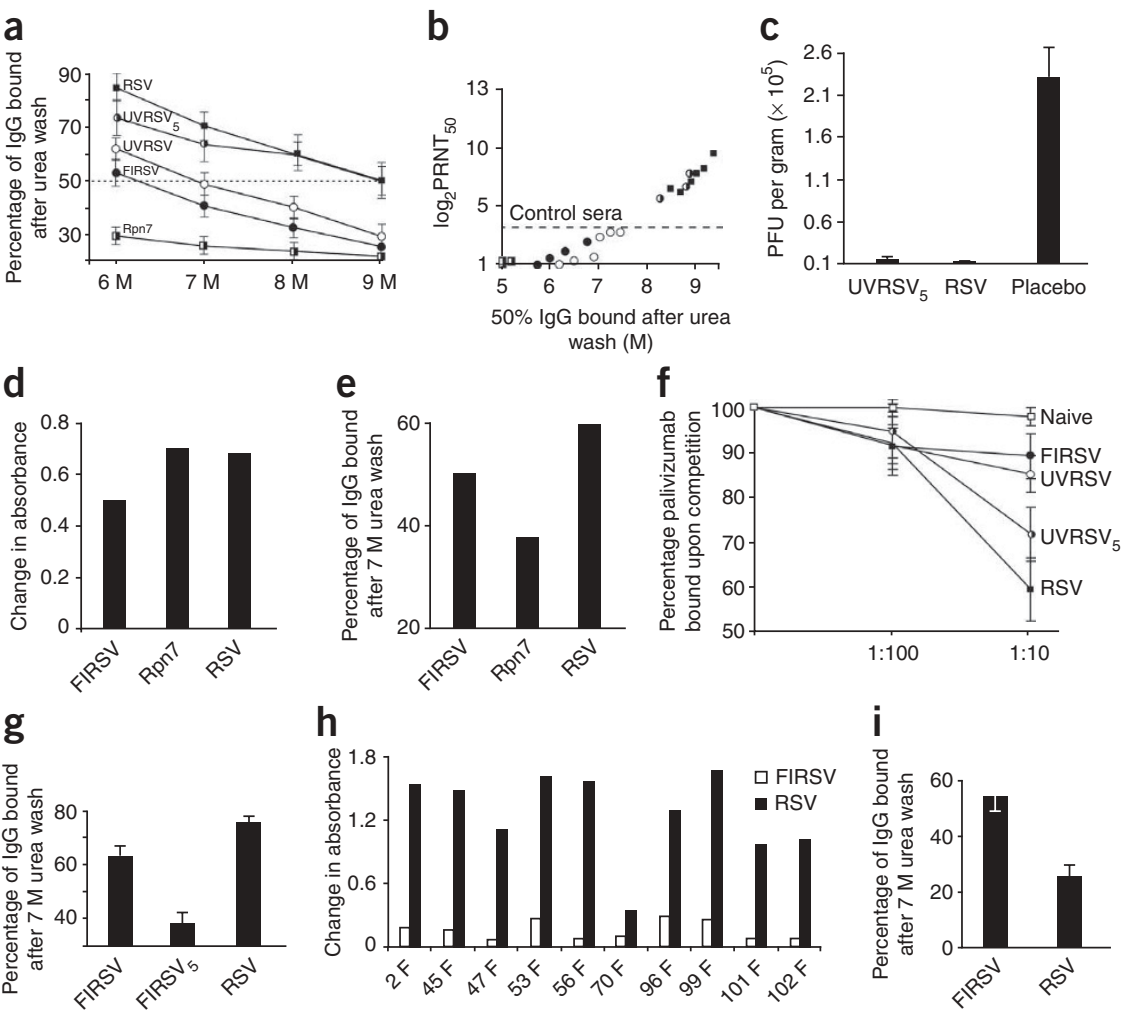

Figure 3 Affinity is crucial for protection against RSV. (a) F protein-specific IgG avidity in sera. $50 \%$ avidity for FIRSV, $P<0.001$ versus RSV, UVRSV $_{5}$ and Rpn7. For UVRSV $5, P<0.05$ versus UVRSV. (b) Correlation between RSVspecific PRNT $_{50}$ and $50 \%$ avidity in sera (prestandardized for $\mathrm{F}$ protein-specific IgG levels). (c) Lung viral titers in recipients of passively transferred sera (prestandardized for $F$ protein-specific IgG levels) from immunized mice. Results are means \pm s.e.m. of three mice per group. For $\mathrm{UVRSV}_{5}, P<0.01$ versus placebo. (d,e) IgG antibody amounts (d) and determination of IgG avidity (e) after a $7 \mathrm{M}$ urea wash against RSV F(422-438) peptide from pooled sera of immunized mice. (f) Binding competition between sera (prestandardized for $F$ protein-specific IgG levels) from immunized mice and palivizumab for RSV $F$ protein. For FIRSV, $P<0.05$ versus naive, UVRSV $_{5}$ and RSV. For UVRSV, $P<0.05$ versus naive, UVRSV 5 and RSV. (g) IgG avidity against $F$ after a $7 \mathrm{M}$ urea wash in mice preimmunized with FIRSV, FIRSV or RSV. (h) Binding of an F protein-specific IgG monoclonal antibody panel 24,48 to FIRSV or RSV. (i) IgG avidity against FIRSV or RSV after a $7 \mathrm{M}$ urea wash in sera from mice preimmunized with FIRSV $_{5}$. Results are means \pm s.e.m. of seven mice per group. $P=0.001$. All data are representative of two to three independent experiments. elicited a short-lived antibody response against $\mathrm{F}$ protein that peaked $45 \mathrm{~d}$ after inoculation, whereas WT RSV elicited a long-lived response to the protein (Fig. 2a).

Subsequently, we compared the neutralizing capacity of antibody elicited by the vaccines. To prevent the confounding effect of steric hindrance in neutralizing antibody responses ${ }^{19,20}$, we standardized all sera for $\mathrm{F}$ protein-specific antibody titers. Antibody elicited by inactivated vaccines lacked neutralizing capacity, whereas WT RSV inoculation generated neutralizing antibody (Fig. 2b).

To compare the quality of the polyclonal antibody response, we characterized the antibody avidities, which have a key role in antibody-mediated protection against other viruses ${ }^{21-23}$. Maturation of avidity was absent in the antibody response elicited by FIRSV or UVRSV, whereas avidity improved over time after inoculation with WT RSV (Fig. 2c,d). Comparison of T helper bias by examining $\mathrm{IgG}_{2 \mathrm{a}} / \mathrm{IgG}_{1}$ ratios of the $\mathrm{F}$ protein-specific antibody responses revealed low $\mathrm{IgG}_{2 \mathrm{a}} / \mathrm{IgG}_{1}$ ratios in nonreplicating vaccine recipients (consistent with $\mathrm{T}_{\mathrm{H}} 2$ bias) and high $\operatorname{IgG}_{2 \mathrm{a}} / \mathrm{IgG}_{1}$ ratios in recipients of WT RSV (Fig. 2e).

\section{Affinity is crucial for protection against RSV}

We then studied the relationship between neutralization and avidity in RSV. For this purpose, we established a mouse model of WT RSV infection that, after resection of the ipsilateral popliteal lymph nodes $7 \mathrm{~d}$ after footpad inoculation, failed to produce high-avidity antibody (Rpn7 mice). In Rpn7 mice, normal amounts of antibody against the F protein were detected after WT RSV inoculation (Supplementary Fig. 2 online), but affinity maturation did not occur (Fig. 3a and Supplementary Fig. 2). Notably, in generating low-avidity antibody, this WT RSV infection was not protective (Fig. 3b).

Subsequently, we promoted maturation of the response to RSV by administering five consecutive daily doses of UVRSV $\left(\mathrm{UVRSV}_{5}\right)$ to

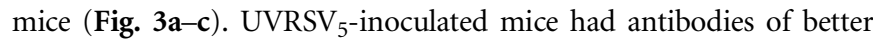
avidity for $\mathrm{F}$ protein than mice given a single dose of UVRSV or FIRSV (Fig. 3a), and these high-avidity antibodies had enhanced neutralizing capacity against RSV (Fig. 3b). We confirmed this correlation between avidity and protection in vivo by comparing passive transfer of sera from mice immunized with WT RSV, UVRSV5 or placebo to naive mice challenged with WT RSV (Fig. 3c). Protection was observed after administration of sera from WT RSV-inoculated and $\mathrm{UVRSV}_{5^{-}}$ inoculated mice.

To rule out the possibility that nonprotective vaccines were eliciting responses against a different repertoire of epitopes than the protective immunogens (rather than failing to elicit maturation against key protective epitopes), we used two complementary strategies. First, we compared antibody responses against a well characterized protective linear epitope in $\mathrm{F}$ protein encompassing amino acids $422-438$ (F(422-438); ref. 24) and found similar amounts of F(422-438)specific antibody in recipients of nonprotective and protective RSV vaccines (Fig. 3d). However, the antibodies elicited by inactivated vaccines were of lower avidity than those detected after WT RSV inoculation (Fig. 3e). Subsequently, we compared the competitive ability of the antibodies elicited by replicating and nonreplicating vaccines against the protective monoclonal antibody palivizumab (Fig. 3f). As expected, owing to their higher avidity for $\mathrm{F}$ protein, sera from mice immunized with WT RSV and $\mathrm{UVRSV}_{5}$ were better able to decrease palivizumab binding to $F$ protein than sera from mice immunized with UVRSV or FIRSV (Fig. 3f). However, all vaccines were able to elicit competitive antibody (Fig. 3f). Taken together, these findings confirm that inactivated vaccines can elicit antibody against crucial protective epitopes in RSV, but the antibody responses are of lower avidity than those elicited by protective vaccines.

Of note, and contrary to our observations in mice immunized with $\mathrm{UVRSV}_{5}$, serial inoculations of FIRSV $\left(\mathrm{FIRSV}_{5}\right)$ worsened antibody 


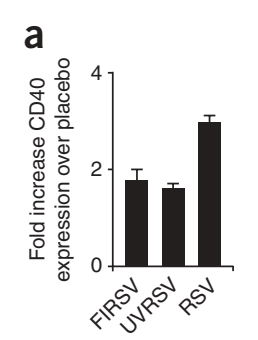

b
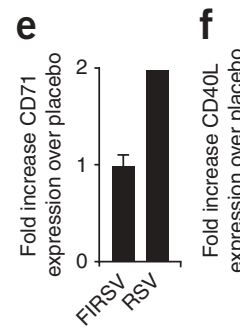

f

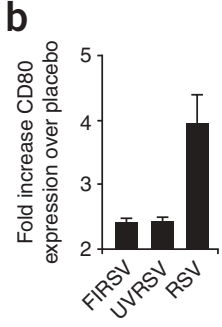

C
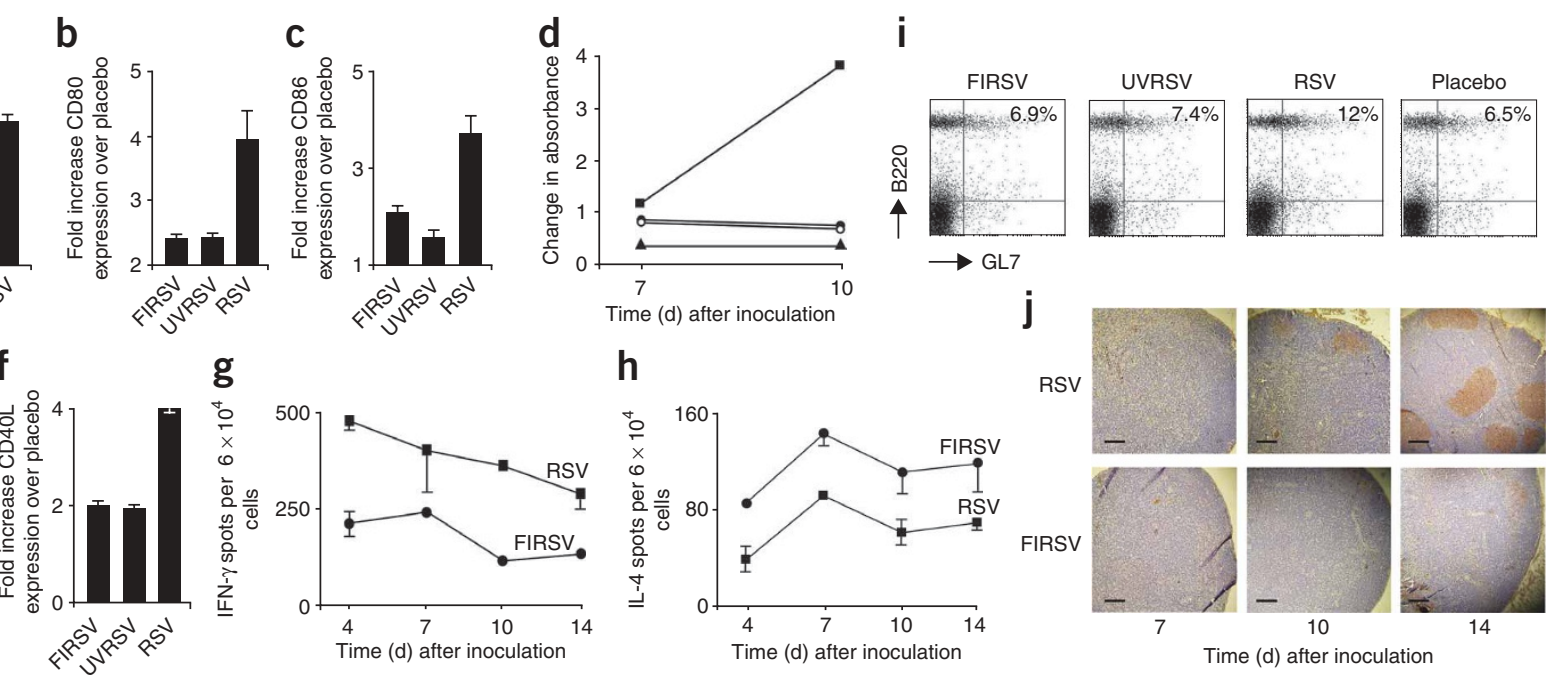

Figure 4 Adaptive immunity after inactivated vaccines. (a-c) Determination of CD40 (a), CD80 (b) and CD86 (c) surface expression in DCs (CD11 $\mathrm{c}^{+}$) from regional popliteal lymph nodes $\left(C C R 7^{+}\right) 24 \mathrm{~h}$ after footpad immunization with FIRSV, UVRSV or RSV (mean fold increase over immunization with placebo of 9-12 mice per group). (d) Lymphoproliferative responses (by BrdU immunoassay) in CD4+ $\mathrm{T}$ lymphocytes from regional popliteal nodes after footpad immunization with FIRSV, UVRSV, RSV or placebo. CD4 ${ }^{+} \mathrm{T}$ lymphocytes were incubated with phytohemagglutinin $(1 \mu \mathrm{g})$ for $48 \mathrm{~h}$. Representative of three independent experiments. (e,f) CD71 (e) and CD40 ligand (f) surface expression in CD4 ${ }^{+} \mathrm{T}$ lymphocytes from regional popliteal nodes by flow cytometry $10 \mathrm{~d}$ after FIRSV, UVRSV or RSV footpad immunization (mean fold increase over immunization with placebo of six mice per group). (g,h) Interferon- $\gamma$ $(\mathrm{IFN}-\gamma ; \mathbf{g})$ and interleukin-4 (IL-4; h) spots per $6 \times 10^{4}$ mononuclear cells from regional popliteal nodes after footpad immunization, as determined by ELISPOT. Results are representative of two independent experiments. (i) Determination of B cell centrocytes $\left(B 220^{+}\right.$and $\left.\mathrm{GL}^{+}\right)$from regional popliteal nodes $14 \mathrm{~d}$ after footpad immunization. Representative of four independent experiments. (j) Germinal center detection (PNA ${ }^{+}$) in slides from regional popliteal nodes after footpad immunization. Scale bar:, $100 \mu \mathrm{m}$. Representative of three independent experiments.

avidity for F protein (Fig. 3g). Therefore, we investigated whether formalin inactivation disrupted RSV epitopes by using a panel of monoclonal antibodies against $\mathrm{F}$ protein and comparing their recognition of RSV and FIRSV (Fig. 3h). Monoclonal antibodies recognized RSV, but binding to FIRSV was comparatively decreased (Fig. 3h). Furthermore, the avidity for FIRSV was enhanced and the avidity for RSV was decreased in sera from mice immunized with FIRSV $_{5}$ (Fig. 3i). These findings suggest that formalin inactivation partially modified RSV epitopes, even though germline antibody elicited by FIRSV recognized protective epitopes in RSV (Figs. 2 and 3d,f) and was associated with ERD pathogenesis ${ }^{5,25}$.

\section{Adaptive immunity after inactivated vaccine administration}

Antibody responses to $\mathrm{T}$ cell-dependent antigens are initiated by dendritic cell (DC) maturation followed by $\mathrm{T}$ helper activation to provide help to B cells, primarily through CD40-CD40 ligand interaction and secretion of cytokines ${ }^{26,27}$. Alternatively, B cells can respond directly to $\mathrm{T}$ cell-dependent antigens upon TLR stimulation $^{27}$. Therefore, we studied DC maturation in mice inoculated with RSV vaccines and found decreased expression of the activation markers CD40, CD80 and CD86 in recipients of inactivated vaccine compared to recipients of live RSV vaccine (Fig. $4 \mathbf{a}-\mathbf{c})$. Similar results were observed in bone marrow-derived DCs incubated with inactivated versus live RSV in vitro, as previously described by other groups $^{28-31}$ (Supplementary Fig. 3 online).

Comparison of $\mathrm{T}$ helper responses revealed decreased $\mathrm{CD}^{+}$ $\mathrm{T}$ lymphocyte proliferation (Fig. 4d) and lower expression of the activation marker CD71 (Fig. 4e) and the co-stimulatory molecules CD40 ligand (Fig. 4f) and inducible co-stimulatory molecule (data not shown) in mice immunized with nonreplicating vaccines. $\mathrm{A}_{\mathrm{H}} 2$ bias characterized by lower interferon- $\gamma$ and higher interleukin- 4 production (Fig. $\mathbf{4 g , h}$ ) was observed in inactivated vaccine recipients.
Upon examination of B cell activation, germinal center formation was similar in recipients of inactivated vaccines and placebo and decreased when compared to recipients of WT RSV (Fig. 4i,j), confirming the absence of affinity maturation.

\section{Deficient activation of TLRs by inactivated vaccines}

TLRs play a crucial part in antibody production ${ }^{26,27}$. In fact, TLR signaling is necessary for DC maturation and $\mathrm{T}$ helper and $\mathrm{B}$ cell activation, all responses that are decreased in mice immunized with inactivated vaccines. Furthermore, TLRs can directly activate B cells to generate antibody against $\mathrm{T}$ cell-dependent antigen ${ }^{27}$. Therefore, we determined whether generation of low-avidity, nonprotective antibody after immunization with inactivated vaccines was associated with deficient TLR activation.

Because promoting antibody affinity maturation with $\mathrm{UVRSV}_{5}$ led to protective responses (Fig. $\mathbf{3 b}, \mathbf{c}$ ), whereas maturation with $\mathrm{FIRSV}_{5}$ polarized responses toward epitopes altered by formalin inactivation (Fig. $3 \mathbf{g}-\mathbf{i}$ ), and because the effects of single doses of both vaccines on the adaptive immune response were similar (Figs. 1-4), we conducted all subsequent experiments with UVRSV as a model of inactivated vaccine immunization.

First, we examined TLR activation in DCs by comparing inhibitor of $\kappa \mathrm{B}-\alpha(\mathrm{I} \kappa \mathrm{B}-\alpha)$ degradation in $\mathrm{CD} 11 \mathrm{c}^{+}$dendritic cells from regional draining lymph nodes (Fig. 5a). Degradation of $\mathrm{I} \kappa \mathrm{B}-\alpha$, a consequence of TLR activation, was decreased in mice inoculated with UVRSV compared to those inoculated with WT RSV (Fig. 5a). Second, to determine whether TLR activation is necessary for maturation of avidity and protection against RSV, we compared responses elicited by

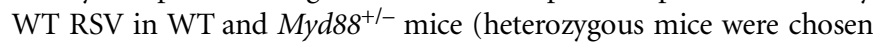
to reflect decreased TLR activation, Fig. $\mathbf{5 b}, \mathbf{c}$ ). MyD88 is a downstream adaptor of most TLRs, including TLR4 and TLR7, which are activated by the F protein and the RSV genome, respectively ${ }^{32-35}$. After 
Figure 5 Deficient activation of TLRs by inactivated vaccines. (a) $I \kappa B-\alpha$ expression by western blotting of DCs $\left(\mathrm{CD} 11 \mathrm{c}^{+}\right)$isolated from regional popliteal lymph nodes $24 \mathrm{~h}$ after footpad inoculation of UVRSV or RSV.

Representative of two independent experiments. (b,c) Determination of $50 \%$ avidity against RSV F protein (b) and RSV-specific neutralization (PRNT $50 ; \mathrm{c}$ ) in sera from WT B6 and $\mathrm{Myd} 8^{+/-}$ mice inoculated intranasally with RSV. Results are means \pm s.e.m. of six to eight mice per group and representative of two independent experiments. $P<0.05$ for both comparisons. (d,e) Determination of $50 \%$ avidity against RSV $F$ protein (d) and RSV-specific neutralization $\left(\mathrm{PRNT}_{50} ; \mathrm{e}\right)$ in sera from BALB/c mice preimmunized with RSV, UVRSV, UVRSV and LPS and UVRSV, LPS, poly $(I: C)$ and polyU. For UVRSV, $P<0.05$ versus UVRSV, LPS, poly $(\mathrm{I}: \mathrm{C})$ and $\mathrm{PolyU}$, and $P<0.01$ versus RSV. Results are representative of two independent experiments. ( $\mathbf{f}-\mathbf{h}) \mathrm{F}$ proteinspecific IgG responses, IgG avidity and

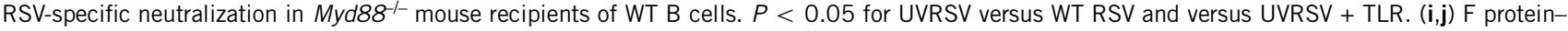
specific IgG responses and neutralization in $\mu \mathrm{MT}$ mouse recipients of $M y d 88^{-1-} \mathrm{B}$ cells.
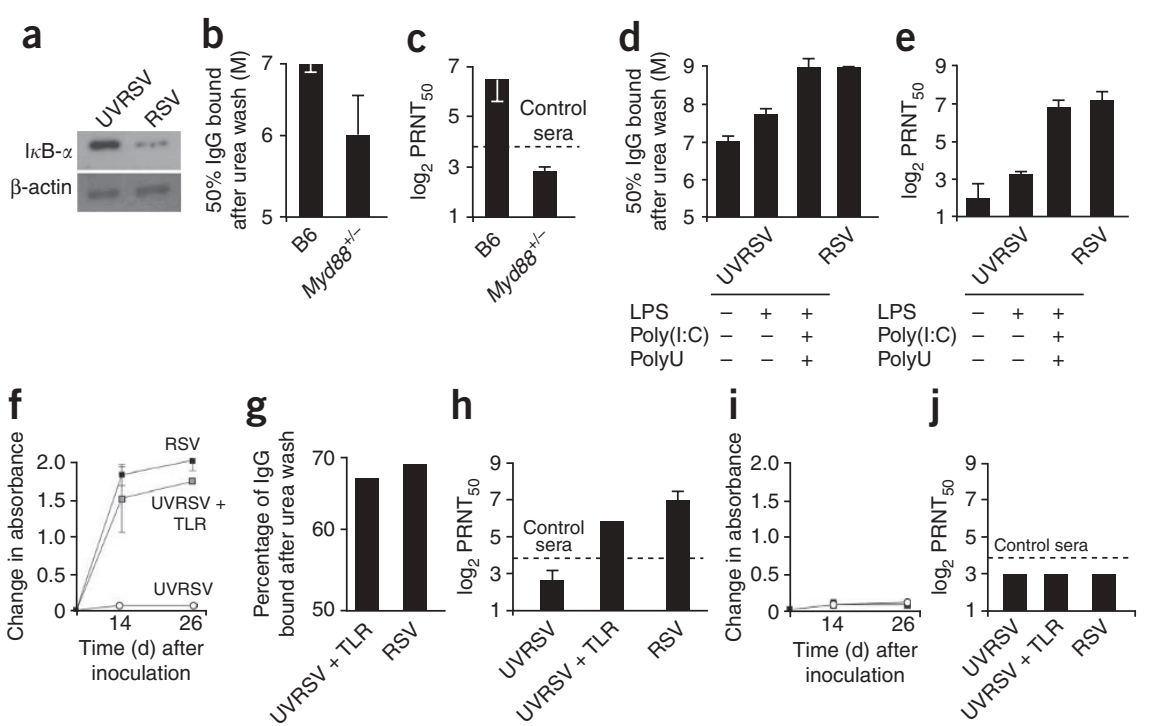

WT RSV i.n. inoculation, both the avidity for $\mathrm{F}$ protein and the neutralizing capacity against RSV were decreased in $M y d 88^{+/-}$mice compared to WT mice (Fig. 5b,c).

Next, we determined whether supplemental TLR stimulation in mice immunized with UVRSV elicited protective antibody against the virus (Supplementary Fig. 4 online). Supplementation of UVRSV with the TLR4 agonist lipopolysaccharide (LPS) increased CD40, CD80 and CD86 cell surface expression in CD11c ${ }^{+}, \mathrm{CCR}^{+}$cells (Supplementary Fig. 4). Moreover, LPS supplementation enhanced germinal center formation in regional lymph nodes (Supplementary Fig. 4), promoted maturation of avidity against F protein (Fig. 5d) and, consequently, promoted protective antibody against RSV (Fig. 5e). Notably, simultaneous stimulation of TLR4, TLR3 and TLR7 with LPS, poly(I:C) and polyU (UVRSV + TLR treatment) further improved affinity maturation and neutralizing capacity in UVRSV recipients (Figs. 5d,e). This positive modulation of protective antibody responses contrasted with the lack of effect observed after adding the TLR-independent adjuvant alum along with UVRSV ${ }^{36,37}$ (Supplementary Fig. 5 online). Taken together, these findings confirm the importance of TLR stimulation in generating affinity maturation and protective antibody responses against RSV.

Finally, to define the requirements for antibody production against RSV and to examine the role of B cells, we passively transferred B cells

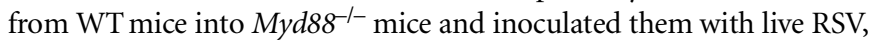
UVRSV + TLR or UVRSV (Fig. $\mathbf{5} \mathbf{f}-\mathbf{j}$ ). Both live RSV and UVRSV + TLR treatments elicited protective antibodies of similar avidity for F protein from WT B cells in $M y d 88^{-1-}$ recipients (Fig. 5f-h). Inoculation with UVRSV failed to elicit a response (Fig. 5f). We passively transferred B cells from $M y d 88^{-/-}$mice to $\mu$ MT mice (lacking mature B cells) and repeated the inoculation strategies (Fig. 5i,j). In this case, $M y d 88^{-/-}$B cells failed to produce RSV-specific IgG in $\mu \mathrm{MT}$ mice, despite the presence of WT DCs (Fig. 5i,j). These findings suggest that stimulation of TLR in B cells is necessary to elicit protective antibody against RSV.

Treatment with UVRSV plus TLR agonists protects against ERD Finally, we examined whether UVRSV + TLR treatment prevented ERD by inoculating mice with UVRSV + TLR, UVRSV, protective live
Figure 6 UVRSV plus TLR agonists protects against ERD. (a) Airway resistance $7 \mathrm{~d}$ after RSV challenge in previously immunized BALB/c mice. Results are means \pm s.e.m. of four to six mice per group and are representative of two independent experiments. Mice were inoculated with WT RSV, UVRSV + TLR, UVRSV or placebo (white squares). For UVRSV + TLR, $P<0.05$ versus UVRSV. $\mathrm{Rn}$, airway resistance.

(b) Pulmonary histopathology $7 \mathrm{~d}$ after RSV challenge in mice that had received UVRSV + TLR preimmunization, including H\&E staining, H\&CR staining and PAS staining showing absence of pneumonia, eosinophilia and mucus production. Scale bar for H\&E staining and PAS staining, $100 \mu \mathrm{m}$; scale bar for H\&CR staining,

$25 \mu \mathrm{m}$. (c) Lung viral titers $4 \mathrm{~d}$ after RSV challenge in recipients of UVRSV + TLR, UVRSV, RSV or placebo. Results are means \pm s.e.m. of four or five mice per group. For UVRSV + TLR, $P<0.05$ versus UVRSV and placebo. (d) Pulmonary histopathology in $\mu$ MT mice immunized with FIRSV and passively transfused with sera obtained from UVRSV + TLR, UVRSV or WT RSV inoculated mice. $\mu$ MT mice were subsequently challenged with WT RSV (day 7 after challenge). Scale bar for H\&E staining, $100 \mu \mathrm{m}$; scale bar for H\&CR staining, $25 \mu \mathrm{m}$. Yellow arrows show eosinophils.
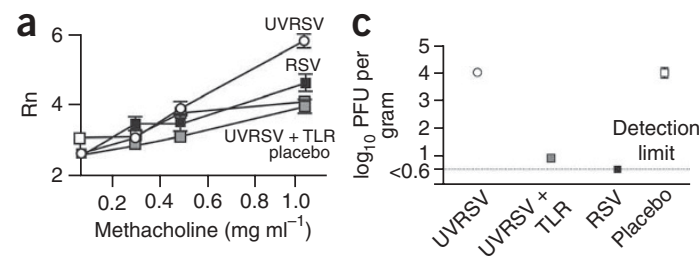

b
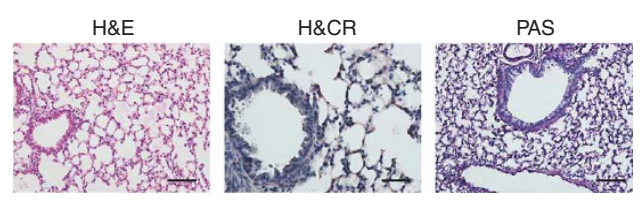

d

d HRE UVRSV
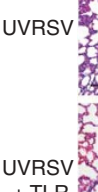

+ TLR

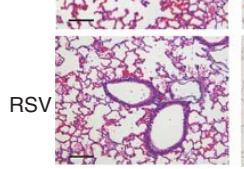
$\mathrm{H} \& \mathrm{CR}$

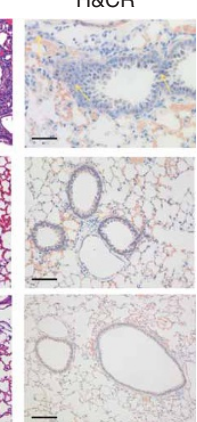


RSV or placebo. After RSV challenge, recipients of UVRSV + TLR had lower airway resistance, milder peribronchiolar and perivascular cellular infiltration and lower eosinophil pulmonary counts and mucus production than UVRSV-immunized mice with ERD (Figs. 6a,b and 1b). RSV lung titers in mice protected by UVRSV + TLR were significantly lower than in mice inoculated with UVRSV (Fig. 6c). In fact, airways reactivity and pulmonary changes in UVRSV + TLR recipients were similar to those observed in mice protected by a previous dose of WT RSV (Figs. 6a-c and 1b).

These observations were confirmed in FIRSV-immunized $\mu \mathrm{MT}$ mice receiving sera from UVRSV + TLR, UVRSV or WT RSV vaccinees (Fig. 6d). Upon RSV challenge, characteristic lung infiltration and eosinophila were present only in recipients of UVRSV sera (Fig. 6d).

\section{DISCUSSION}

The mechanism responsible for the lack of protection by the antibody response elicited by FIRSV against RSV remained unclear for decades, hampering development of new vaccines against the virus. This study demonstrates that the nonprotective antibody response elicited by inactivated RSV immunogens, and ultimately associated with the development of $\mathrm{ERD}^{4,5}$, results from lack of affinity maturation due to deficient TLR activation in B cells. These findings modify the previous paradigm, ascribing poor antibody function to formalin disruption of protective epitopes $7,13,14$. They also open the possibility that inactivated RSV vaccines may be rendered safe and effective by inclusion of TLR agonists in their formulation.

Several observations support the importance of antibody avidity for protection against respiratory viruses, including measles virus with its risk for atypical disease ${ }^{21,38}$. A formalin-inactivated vaccine against measles virus elicited low-avidity, nonprotective antibody associated with serious clinical manifestations in individuals exposed to WT measles virus ${ }^{21}$. Low-avidity antibody neutralized viral infection through the CD46 low-affinity measles virus receptor, but not the CD150 high-affinity WT measles virus receptor, and promoted immune complex-mediated illness ${ }^{21}$. However, low-avidity polyclonal responses against other viruses may suffice to confer protection. For example, disease enhancement has never been described for influenza virus, despite the wide use of protective nonreplicating vaccines against influenza that do not promote affinity maturation ${ }^{39}$. The low-affinity interaction between the binding site for the receptor in the influenza hemagglutinin and the cellular receptor for the virus $\left(K_{\mathrm{d}}=2 \times 10^{-3}\right.$; ref. 40$)$ may facilitate neutralization by antibodies of relatively low avidity. In the case of RSV, differences in affinity affect the neutralizing capacity of RSV-specific monoclonal antibodies ${ }^{15}$, and our study provides evidence for their crucial role in natural protection against WT infection. The WT receptor for RSV has not been identified. Perhaps differences in the affinity of the interaction between viral attachment proteins and their receptors or in the mechanisms of neutralization among viruses may explain the discrepancies in the efficacies of inactivated vaccines against RSV and influenza ${ }^{23,41,42}$.

This report highlights the importance of TLR activation in B cells for protection against RSV and prevention of ERD. RSV F protein interacts with TLR4, initiating membrane fusion and MyD88dependent and MyD88-independent pathways leading to activation of several transcription factors, notably nuclear factor- $\kappa \mathrm{B}$. Once RSV begins transcribing and replicating its genome, TLR-mediated detection seems to depend upon autophagy-associated mechanisms that engage TLR7 and TLR3 in endosomal compartments (cytosolic viral double-stranded RNA also activates cytoplasmic caspase-recruiting domain helicases and RNA-dependent protein kinase ${ }^{33-35,43-45}$ ). Our data suggest that efficient detection of RSV by TLRs, which converge on a redundant set of transcription factors, is necessary for antibody production and affinity maturation. Nonreplicating RSV vaccines (for example, UVRSV) stimulate only a subset of these receptors (such as TLR4; ref. 31) in a weak fashion, becoming protective only after incorporation of exogenous TLR ligands. Because most TLRs share the same downstream effectors, different agonists may achieve similar effects provided that the intensity of the signal is appropriate. This conclusion is supported by the synergistic effect of adjuvants containing mixed TLR ligands. Remediation of UVRSV with TLR agonists leads to DC maturation, $\mathrm{T}$ helper activation and $\mathrm{B}$ cell affinity maturation. Specifically, our B cell transfer experiments and those using $M y d 88^{+/-}$mice suggest the existence of a threshold of activation below which affinity maturation does not occur and protective antibodies are not elicited. Moreover, for WT RSV the threshold can only be surpassed if MyD88-dependent pathways in B cells are engaged. This does not imply that MyD88-independent pathways do not have a role, but rather that their activation alone is insufficient.

Perhaps a consequence of the use of formalin as the chemical for vaccine inactivation in 1966 was that it biased the $\mathrm{T}$ cell response in ERD toward $\mathrm{T}_{\mathrm{H}} 2$ (ref. 7). Partial alteration of RSV epitopes during formalin inactivation probably contributed to the generation of nonprotective antibody. But epitope disruption had a secondary role in disease priming, as the low-avidity antibodies elicited by FIRSV recognized protective epitopes, and other nonreplicating RSV vaccines not treated with formalin in this study and in other publications showed evidence of priming for aberrant immune manifestations ${ }^{6,11,12}$. Other studies associated the $\mathrm{T}_{\mathrm{H}} 2$ bias in ERD to the absence of $\mathrm{T}$ helper-modulatory cytotoxic $\mathrm{T}$ lymphocytes in FIRSV recipients ${ }^{8,46}$ or postulated a role for alum in $\mathrm{T}$ cell polarization ${ }^{47}$. However, ERD phenotypes did not differ with or without alum ${ }^{5,6}$. Whether deficient TLR activation contributed to the $\mathrm{T}_{\mathrm{H}} 2$ bias in ERD remains to be determined.

Notably, our findings may also help clarify why none of the children who were seropositive for RSV before immunization with FIRSV developed $\mathrm{ERD}^{4}$, even though WT RSV infections confer only partial protection against subsequent exposures ${ }^{1,2}$. The preexisting highavidity antibodies elicited by WT RSV before immunization probably outcompeted the low-avidity clones elicited by FIRSV and prevented nonprotective, pathogenic B cell priming against the virus. Our study also may explain why no child ever developed ERD twice, as the B cells elicited by RSV infection during ERD also eventually outcompeted pathogenic B cells primed by FIRSV and reestablished a normal response against future re-infections.

In summary, we show a key role for antibody avidity in protective responses against RSV and in the pathogenesis of ERD. Poor TLR stimulation by inactivated RSV vaccines was associated with lack of maturation and led to production of nonprotective antibodies. These antibodies were essential for ERD pathogenesis, as they failed to neutralize RSV, allowing unrestricted replication and secondary stimulation of FIRSV-primed $\mathrm{T}_{\mathrm{H}} 2$ cells. Furthermore, low-avidity antibody contributed to disease severity through immune complex formation and deposition in affected tissue ${ }^{5}$. Our findings indicate that safe and effective RSV vaccines for infants will require neutralizing antibodies with similar avidity for protective antigens to that elicited by live virus inoculation.

\section{METHODS}

Viruses and vaccines. We prepared FIRSV vaccines with the RSV A2 strain grown in epidermoid laryngeal carcinoma Hep- 2 cells ${ }^{5}$ and achieved ultraviolet 
inactivation of RSV A2 through three cycles of irradiation at a 3,600 $\times$ $100 \mu \mathrm{J} \mathrm{cm}^{-2}$ dose of ultraviolet light. We obtained PFP from RSV A2-infected Hep-2 cells.

Mice and immunizations. We housed 4-8-week-old BALB/c and C57BL/6 mice, $\mu \mathrm{MT}$ mice (Jackson Laboratories), $M y d 88^{+/-}$and $M y d 88^{-/-}$mice under laminar flow hoods in an environmentally controlled specific pathogen-free animal facility. We performed footpad immunizations with $50 \mu$ l containing $1 \times 10^{5}$ plaque-forming units (PFU) of RSV, FIRSV or UVRSV or $1 \mu \mathrm{g}$ of either PFP or Hep-2 lysates (placebo). We immunized the mice i.n. with $1 \times 10^{5} \mathrm{PFU}$ RSV . For RSV i.n. challenge of preimmunized mice, we used $1 \times 10^{6} \mathrm{PFU}$ of live RSV. We administered TLR agonists by footpad inoculation as follows: LPS (Sigma), $1 \mu \mathrm{g}$; poly(I:C) (Invivogen), $2.5 \mu \mathrm{g}$; and polyU (Invivogen), $0.1 \mu \mathrm{g}$. All experiments were approved by and performed according to guidelines of the Animal Care and Use Committee from the Johns Hopkins Medical Institutions and the Infant Foundation.

Airway hyperresponsiveness. Seven days after challenge, we anesthetized the mice $20 \mathrm{ml} \mathrm{kg}^{-1}$ of Avertin (2, 2, 2-tribromoethanol, Fluka + 2-methyl-2butanol, Fisher), intubated them and ventilated them at a rate of 120 breaths per min with a constant tidal volume of air $(0.2 \mathrm{ml})$. We then paralyzed the mice with decamethonium bromide $\left(25 \mu \mathrm{g} \mathrm{kg}^{-1}\right)$ and, after establishing a stable airway pressure, gave them acetylcholine intravenously $\left(50 \mu \mathrm{g} \mathrm{kg}^{-1}\right)$. We measured the dynamic airway pressure for $5 \mathrm{~min}$. We confirmed airway pressure time index determinations by analysis of lung resistance to aerosolized methacholine $\left(0.01-1 \mathrm{mg} \mathrm{kg}^{-1}\right)$.

Histopathology. We stained formalin-fixed lung sections $(5-7 \mu \mathrm{m})$ with either periodic acid-Schiff, hematoxylin and Congo Red or H\&E. We scored eosinophils as previously described ${ }^{25}$ and we evaluated pneumonia as described in the Supplementary Methods.

We removed popliteal lymph nodes aseptically from mice and sliced them in 5-7- $\mu \mathrm{m}$ sections (after fixation) for immunohistochemistry. We used a lectin from Arachis hypogaea (PNA) conjugated with biotin (Sigma) to detect centrocytes.

Virus titration in lung tissue. We aseptically removed lungs from mice $4 \mathrm{~d}$ after RSV challenge and obtained titers as described elsewhere ${ }^{5}$. Results are expressed as PFU g ${ }^{-1}$ of lung tissue.

Antibody assays. We measured avidity with $6,7,8$ or $9 \mathrm{M}$ urea washes, as previously described ${ }^{21}$. We standardized sera for avidity-neutralizing antibody correlations, competition binding assays, and passive transfer experiments for F protein-specific antibody levels before use. For competition assays, we used palivizumab at a dose of $1 \mathrm{ng}$ per well. We performed RSV-specific neutralization with volumes of $200 \mu \mathrm{l}$ per sample. We assayed sera from uninfected mice as controls.

Passive transfer experiments. We gave BALB/c mice a maximum of $200 \mu \mathrm{l}$ of sera containing equal amounts of $\mathrm{F}$ protein-specific antibody via tail vein injection. The control group received $200 \mu \mathrm{l}$ of naive sera. One day later, we inoculated all mice i.n. with $1 \times 10^{6} \mathrm{PFU}$ of WT RSV. Four days after infection, we removed the lungs and performed virus titration as described above. For B cell transfer experiments, we injected $7 \times 10^{6}$ purified B cells from C57BL/6 or $M y d 88^{-/-}$mice intravenously into $\mathrm{Myd} d 88^{-/-}$or $\mu \mathrm{MT}$ mice, respectively.

Flow cytometry. We stained cells for flow cytometry by standard procedures and used antibody to mouse CD11c (BD Biosciences), CCR7 (BioLegend), $\mathrm{CD} 40, \mathrm{CD} 80$ and CD86 (all from BD Biosciences) to characterize DC activation. We used antibodies to mouse CD4 and CD154 (BD Biosciences) for $\mathrm{T}$ cell analyses. We detected germinal center cells with antibody to mouse CD45R (B220) and the B cell activation antigen GL7 (BD Biosciences). We evaluated cells with FACScalibur (BD Biosciences) and analyzed them with CELLQuest software (BD Biosciences).

Lymphoproliferation assay. We isolated $\mathrm{CD} 4^{+} \mathrm{T}$ lymphocytes from popliteal nodes and incubated them for $48 \mathrm{~h}$ in the presence of phytohemagglutinin. Then, we added a pulse of $\mathrm{BrdU}$ ( $10 \mu \mathrm{M}$, Roche) to each well for the last $18 \mathrm{~h}$ of the assay and detected proliferation by ELISA (Roche).
Enzyme-linked immunosorbent spot assays. We performed enzyme-linked immunosorbent spot (ELISPOT) assays in freshly obtained lymph node cells as previously described ${ }^{25}$.

Western blots. We obtained purified CD11c cells (Miltenyi Biotec) from popliteal nodes of mice belonging to various experimental groups and detected

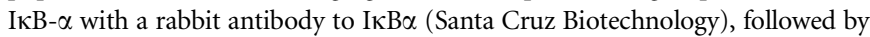
a horseradish peroxidase-conjugated antibody to rabbit IgG (Santa Cruz Biotechnology) and SuperSignal Chemiluminescent Substrate (Pierce).

$\mathrm{CD8}^{+} \mathrm{T}$ lymphocyte depletion. We depleted $\mathrm{CD} 8^{+} \mathrm{T}$ lymphocytes by administration of Gk2.43 monoclonal antibody, as previously described ${ }^{18}$. We used an irrelevant monoclonal antibody as a control.

Statistical analyses. We analyzed data with statistical software (GraphPad Prism). We made comparisons among airway responses by analysis of variance for parametric cases. We used the Kruskal-Wallis test for nonparametric comparisons where appropriate. We analyzed maturation of avidity after immunization by regression analysis. Data are expressed as means \pm s.e.m. $P<0.05$ was considered significant.

Note: Supplementary information is available on the Nature Medicine website.

\section{ACKNOWLEDGMENTS}

PFP was a generous gift from V. Randolph (Wyeth Lederle) and Gk2.43 monoclonal antibody for $\mathrm{CD}^{+} \mathrm{T}$ lymphocyte depletion was generously provided by B. Graham and T. Johnson (National Institute of Allergy and Infectious Diseases, US National Institutes of Health). We thank M. del Carmen Puggioli for excellent technical assistance. This work was supported by AI-054952 (F.P.P.) and the Thomas and Carol McCann Innovative Research Fund for Asthma and Respiratory Diseases (F.P.P.). M.F.D., A.C.M., J.P.B. and S.C. are recipients of Doctoral Awards from the Consejo Nacional de Investigaciones Científicas y Técnicas, Argentina. G.A.M. is a recipient of the Thrasher Research Fund Early Career Award.

\section{AUTHOR CONTRIBUTIONS}

M.F.D., S.C., A.C.M., G.A.M., J.Z.H., J.P.B., L.D., A.T. and H.-Y.C. performed research; J.R., J.A.M. and W.M. contributed new reagents and/or analytic tools; M.F.D., W.M., J.A.M., J.P.B., P.M.I. and F.P.P. analyzed data; F.P.P. designed research; M.F.D., P.M.I. and F.P.P. wrote the paper.

Published online at http://www.nature.com/naturemedicine/

Reprints and permissions information is available online at http://npg.nature.com/ reprintsandpermissions/

1. Collins, P.L. et al. Fields Virology. (eds. Knipe, D.M. \& Howley, P.M.) 1443-1486 (Raven Press, New York, 2001).

2. Glezen, W.P., Taber, L.H., Frank, A.L. \& Kasel, J.A. Risk of primary infection and reinfection with respiratory syncytial virus. Am. J. Dis. Child. 140, 543-546 (1986).

3. Shay, D.K. et al. Bronchiolitis-associated hospitalizations among US children, 1980-1996. J. Am. Med. Assoc. 282, 1440-1446 (1999).

4. Kim, H.W. et al. Respiratory Ssyncytial virus disease in infants despite prior administration of antigenic inactivated vaccine. Am. J. Epidemiol. 89, 422-434 (1969).

5. Polack, F.P. et al. A role for immune complexes in enhanced respiratory syncytial virus disease. J. Exp. Med. 196, 859-865 (2002).

6. Graham, B.S. et al. Priming immunization determines $T$ helper cytokine mRNA expression patterns in lungs of mice challenged with respiratory syncytial virus. J. Immunol. 151, 2032-2040 (1993).

7. Moghaddam, A. et al. A potential molecular mechanism for hypersensitivity caused by formalin-inactivated vaccines. Nat. Med. 12, 905-907 (2006).

8. Srikiatkhachorn, A. \& Braciale, T.J. Virus-specific $C D 8^{+}$T lymphocytes downregulate T helper cell type 2 cytokine secretion and pulmonary eosinophilia during experimental murine respiratory syncytial virus infection. J. Exp. Med. 186, 421-432 (1997).

9. Connors, M. et al. Pulmonary histopathology induced by respiratory syncytial virus (RSV) challenge of formalin-inactivated RSV-immunized BALB/c mice is abrogated by depletion of CD4+ T cells. J. Virol. 66, 7444-7451 (1992).

10. Connors, M. et al. Enhanced pulmonary histopathology induced by respiratory syncytial virus (RSV) challenge of formalin-inactivated RSV-immunized BALB/c mice is abrogated by depletion of interleukin-4 (IL-4) and IL-10. J. Virol. 68, 5321-5325 (1994).

11. Connors, M. et al. Cotton rats previously immunized with a chimeric RSV FG glycoprotein develop enhanced pulmonary pathology when infected with RSV, a phenomenon not encountered following immunization with vaccinia-RSV recombinants or RSV. Vaccine 10, 475-484 (1992).

12. Murphy, B.R., Sotnikov, A.V., Lawrence, L.A., Banks, S.M. \& Prince, G.A. Enhanced pulmonary histopathology is observed in cotton rats immunized with 
formalin-inactivated respiratory syncytial virus (RSV) or purified $F$ glycoprotein and challenged with RSV 3-6 months after immunization. Vaccine 8, 497-502 (1990).

13. Murphy, B.R. \& Walsh, E.E. Formalin-inactivated respiratory syncytial virus vaccine induces antibodies to the fusion glycoprotein that are deficient in fusion-inhibiting activity. J. Clin. Microbiol. 26, 1595-1597 (1988).

14. Prince, G.A. et al. Enhancement of respiratory syncytial virus pulmonary pathology in cotton rats by prior intramuscular inoculation of formalin-inactivated virus. J. Virol. 57, 721-728 (1986)

15. Wu, H. et al. Development of motavizumab, an ultra-potent antibody for the prevention of respiratory syncytial virus infection in the upper and lower respiratory tract. J. Mol. Biol. 368, 652-665 (2007).

16. Johnson, $\mathrm{S}$. et al. A direct comparison of the activities of two humanized respiratory syncytial virus monoclonal antibodies: MEDI-493 and RSHZI9. J. Infect. Dis. 180, 35-40 (1999)

17. American Academy of Pediatrics Committee on Infectious Diseases and Committee on Fetus and Newborn. Revised indications for the use of palivizumab and respiratory syncytial virus immune globulin intravenous for the prevention of respiratory syncytial virus infections. Pediatrics 112, 1442-1446 (2003).

18. Graham, B.S. et al. Role of T lymphocyte subsets in the pathogenesis of primary infection and rechallenge with respiratory syncytial virus in mice. J. Clin. Invest. 88, 1026-1033 (1991).

19. Murphy, B.R et al. Immunization of cotton rats with the fusion (F) and large G) glycoproteins of respiratory syncytial virus ( (RSV) protects against RSV challenge without potentiating RSV disease. Vaccine 7, 533-540 (1989).

20. Mapletoft, J.W. et al. Intranasal immunization of mice with a formalin-inactivated bovine respiratory syncytial virus vaccine co-formulated with $\mathrm{CpG}$ oligodeoxynucleotides and polyphosphazenes results in enhanced protection. J. Gen. Virol. 89, 250-260 (2008)

21. Polack, F.P., Hoffman, S.J., Crujeiras, G. \& Griffin, D.E. A role for nonprotective complement-fixing antibodies with low avidity for measles virus in atypical measles. Nat. Med. 9, 1209-1213 (2003).

22. Bachmann, M.F. et al. The role of antibody concentration and avidity in antiviral protection. Science 276, 2024-2027 (1997)

23. Fleury, D. et al. A complex of influenza hemagglutinin with a neutralizing antibody that binds outside the virus receptor binding site. Nat Struct. Biol. 6, $530-534$ (1999).

24. Arbiza, J. et al. Characterization of two antigenic sites recognized by neutralizing monoclonal antibodies directed against the fusion glycoprotein of human respiratory syncytial virus. J. Gen. Virol. 73, 2225-2234 (1992).

25. Melendi, G.A. et al. C5 modulates airway hyperreactivity and pulmonary eosinophilia during enhanced respiratory syncytial virus disease by decreasing $\mathrm{C} 3 \mathrm{a}$ receptor expression. J. Virol. 81, 991-999 (2007).

26. Medzhitov, R. Toll-like receptors and innate immunity. Nat. Rev. Immunol. 1, 135-145 (2001).

27. Pasare, C. \& Medzhitov, R. Control of B-cell responses by Toll-like receptors. Nature 438, 364-368 (2005).

28. Rudd, B.D. et al. Type I interferon regulates respiratory virus infected dendritic cell maturation and cytokine production. Viral Immunol. 20, 531-540 (2007).

29. Rudd, B.D. et al. MyD88-mediated instructive signals in dendritic cells regulate pulmonary immune responses during respiratory virus infection. J. Immunol. 178 , 5820-5827 (2007).
30. Smit, J.J. et al. The balance between plasmacytoid DC versus conventional DC determines pulmonary immunity to virus infections. PLoS ONE 3, e1720 (2008).

31. Polack, F.P. et al. The cysteine-rich region of respiratory syncytial virus attachment protein inhibits innate immunity elicited by the virus and endotoxin. Proc. Natl. Acad. Sci. USA 102, 8996-9001 (2005).

32. Kawai, T., Adachi, O., Ogawa, T., Takeda, K. \& Akira, S. Unresponsiveness of MyD88deficient mice to endotoxin. Immunity 11, 115-122 (1999).

33. Kurt-Jones, E.A. et al. Pattern recognition receptors TLR4 and CD14 mediate response to respiratory syncytial virus. Nat. Immunol. 1, 398-401 (2000).

34. Rudd, B.D. et al. Deletion of TLR3 alters the pulmonary immune environment and mucus production during respiratory syncytial virus infection. J. Immunol. 176 1937-1942 (2006)

35. Lindemans, C.A. et al. Respiratory syncytial virus inhibits granulocyte apoptosis through a phosphatidylinositol 3-kinase and NF-kB-dependent mechanism. J. Immunol. 176, 5529-5537 (2006).

36. Gavin, A.L. et al. Adjuvant-enhanced antibody responses in the absence of Toll-like receptor signaling. Science 314, 1936-1938 (2006).

37. Kool, M. et al. Cutting Edge: alum adjuvant stimulates inflammatory dendritic cells through activation of the NALP3 inflammasome. J. Immunol. 181, 3755-3759 (2008).

38. Polack, F.P. et al. Production of atypical measles in rhesus macaques: evidence for disease mediated by immune complex formation and eosinophils in the presence of fusion-inhibiting antibody. Nat. Med. 5, 629-634 (1999)

39. Brokstad, K.A. et al. Cross-reaction but no avidity change of the serum antibody response after influenza vaccination. Vaccine 13, 1522-1528 (1995).

40. Knossow, M. et al. Mechanism of neutralization of influenza virus infectivity by antibodies. Virology 302, 294-298 (2002).

41. Barbey-Martin, C. et al. An antibody that prevents the hemagglutinin low pH fusogenic transition. Virology 294, 70-74 (2002).

42. Sauter, N.K. et al. Hemagglutinins from two influenza virus variants bind to sialic acid derivatives with millimolar dissociation constants: a $500-\mathrm{MHz}$ proton nuclear magnetic resonance study. Biochemistry 28, 8388-8396 (1989).

43. Kolokoltsov, A.A. et al. Small interfering RNA profiling reveals key role of clathrin mediated endocytosis and early endosome formation for infection by respiratory syncytial virus. J. Virol. 81, 7786-7800 (2007).

44. Liu, P. et al. Retinoic acid-inducible gene I mediates early antiviral response and Tolllike receptor 3 expression in respiratory syncytial virus-infected airway epithelial cells. J. Virol. 81, 1401-1411 (2007).

45. Bhoj, V.G. et al. MAVS and MyD88 are essential for innate immunity but not cytotoxic T lymphocyte response against respiratory syncytial virus. Proc. Natl. Acad. Sci. USA 105, 14046-14051 (2008).

46. Hussell, T., Baldwin, C.J., O'Garra, A. \& Openshaw, P.J. CD8 ${ }^{+}$T cells control $T_{H^{2}}$ driven pathology during pulmonary respiratory syncytial virus infection. Eur. J. Immunol. 27, 3341-3349 (1997).

47. Neuzil, K.M. et al. Adjuvants influence the quantitative and qualitative immune response in BALB/c mice immunized with respiratory syncytial virus FG subunit vaccine. Vaccine 15, 525-532 (1997).

48. García-Barreno, B. et al. Marked differences in the antigenic structure of human respiratory syncytial virus F and G glycoproteins. J. Virol. 63, 925-932 (1989). 